Article

\title{
Are Total and Individual Dietary Lignans Related to Cardiovascular Disease and Its Risk Factors in Postmenopausal Women? A Nationwide Study
}

\author{
Anna Maria Witkowska ${ }^{1, *(1)}$, Anna Waśkiewicz ${ }^{2}$, Małgorzata Elżbieta Zujko ${ }^{1}$ (D), \\ Danuta Szcześniewska ${ }^{2}$, Urszula Stepaniak ${ }^{3}$, Andrzej Pająk ${ }^{3}$ and Wojciech Drygas ${ }^{2,4}$ \\ 1 Department of Food Biotechnology, Faculty of Health Sciences, Medical University of Bialystok, \\ Szpitalna 37, 15-295 Bialystok, Poland; malgorzata.zujko@umb.edu.pl \\ 2 Department of Epidemiology, Cardiovascular Disease Prevention and Health Promotion, \\ Institute of Cardiology, Alpejska 42, 04-628 Warsaw, Poland; awaskiewicz@ikard.pl (A.W.); \\ dszczesniewska@ikard.pl (D.S.); wdrygas@ikard.pl (W.D.) \\ 3 Department of Epidemiology and Population Studies, Faculty of Health Sciences, Institute of Public Health, \\ Jagiellonian University Medical College, Grzegórzecka 20, 31-531 Krakow, Poland; \\ urszula.stepaniak@uj.edu.pl (U.S.); andrzej.pajak@uj.edu.pl (A.P.) \\ 4 Department of Social and Preventive Medicine, Faculty of Health Sciences, Medical University of Lodz, \\ Hallera 1, 90-001 Lodz, Poland \\ * Correspondence: witam@umb.edu.pl; Tel.: +48-85-686-5088
}

Received: 20 June 2018; Accepted: 2 July 2018; Published: 4 July 2018

\begin{abstract}
The study objectives were to examine total and individual lignan intakes and their dietary sources in postmenopausal Polish women and to investigate the relationship between lignan intake and the prevalence of cardiovascular disease (CVD), hypertension, hypercholesterolemia and central obesity. A total of 2599 postmenopausal women, participants of the Multi-centre National Population Health Examination Surveys (WOBASZ and WOBASZ II) were selected. Of them, 916 had a history of CVD. Nutritional data were collected using a single 24-h dietary recall. Data on lignan content in food, i.e., lariciresinol (LARI), matairesinol (MAT), pinoresinol (PINO) and secoisolariciresinol (SECO), were collected from the available lignan databases. In postmenopausal women, total and individual lignan intakes (SECO, PINO, MAT) were not associated with the prevalence of CVD and its risk factors. The intake of LARI was linked by $30 \%$ to the reduced odds for hypercholestrolemia. This study reinforces the existing concept that dietary total lignans are not associated with the prevalence of CVD, and provides further evidence that they are not linked to CVD risk factors such as hypertension, hypercholesterolemia and central obesity. However, the intake of LARI should be taken into consideration in further studies with regard to its potentially beneficial effect in hypercholesterolemia.
\end{abstract}

Keywords: lignan; lariciresinol; postmenopausal; cardiovascular disease; hypercholesterolemia; hypertension; central obesity

\section{Introduction}

Lignans are plant-derived diphenolic compounds formed by the dimerization of two cinnamic acid residues that are widely distributed in seeds, grains, legumes, fruit and vegetables. The most common food representatives of lignans are secoisolariciresinol, matairesinol, pinoresinol and lariciresinol. Compared to flavonoids and phenolic acids, which are present in food in larger amounts [1], the usual intake of lignans is minor, ranging between $~ 1-1.6 \mathrm{mg} /$ day when these 
four lignans are taken into account [2]. Dietary sources of total and individual lignans may vary among countries and are determined by the habitual patterns of food consumption.

In the human gastrointestinal tract, the enterolignan precursors, i.e., secoisolariciresinol, matairesinol, pinoresinol, lariciresinol, are metabolized by anaerobic bacteria to enterolignans, which demonstrate antioxidant properties as well as estrogenic activity [3,4]. In addition, as it has been recently reviewed, lignans possess anti-aging properties [5]. These characteristics of lignans may be important for the prevention of cardiovascular disease (CVD) in postmenopausal women, who manifest higher prevalence of CVD in contrast to premenopausal females [6]. In the intervention studies, lignan supplements demonstrated lowering effects on plasma lipids [7]. Epidemiological evidence, however, is inconclusive. Some studies suggest potential advantages of habitual intakes of total or individual lignans [8-10]. An inverse association between the high lignan intake and hypertension [8] and the incidence of CVD [9] was found. The intake of an individual lignan, matairesinol, was inversely associated with the mortality due to CVD [10]. Others do not support the concept of reduced mortality due to dietary phytoestrogen intake [11]. Only slight associations of total lignan intakes with reduced blood pressure, lower prevalence of hypertension, lower triglyceride concentration and reduced aortic stiffness have been described [12]. In turn, individual lignans such as matairesinol and secoisolariciresinol have been favorably related to the parameters of vascular inflammation and endothelial dysfunction [13]. These ambiguous findings of the previous studies warrant further research regarding dietary lignan intakes and their presumable beneficial role in CVD.

The current study was designed to assess total and individual lignan intakes and their dietary sources in postmenopausal Polish women, and to assess the relationship between lignan intake and the prevalence of hypercholesterolemia, hypertension, central obesity and cardiovascular disease.

\section{Materials and Methods}

\subsection{Participants}

The Multi-centre National Population Health Examination Surveys, WOBASZ (2003-2005) and WOBASZ II (2013-2014) were the two largest population-based cross-sectional studies carried out in Poland by the National Institute of Cardiology, Warsaw, Poland, in collaboration with five Polish medical universities [14-16]. These surveys evaluated representative random samples of the total Polish population in 21,000 men and women aged 20-74 years (WOBASZ) and 20+ years. (WOBASZ II). All subjects gave their informed consent for inclusion before they participated in the studies. The studies were conducted in accordance with the Declaration of Helsinki, and the protocol was approved by the Ethics Committee of the Institute of Cardiology (WOBASZ, No. 708) and (WOBASZ II, No. 1344). Nutrition studies were conducted in the approximately $62 \%$ sample of the study population (Figure 1). From these participants, 2599 postmenopausal women were selected based on the question referring to the occurrence of natural menopause and completed records. The same menopause criterion of six months from the termination of menstruation was adopted in both studies. The exclusion criteria were pregnancy and surgical menopause. Then, a group of 916 women with a history of CVD was extracted from the general study group of postmenopausal women. The remaining 1683 women without past history of CVD served as controls. Data on hypertension, hypercholesterolemia and obesity were obtained for all 2599 women. The flow-chart of the study participants is provided in Figure 1. 


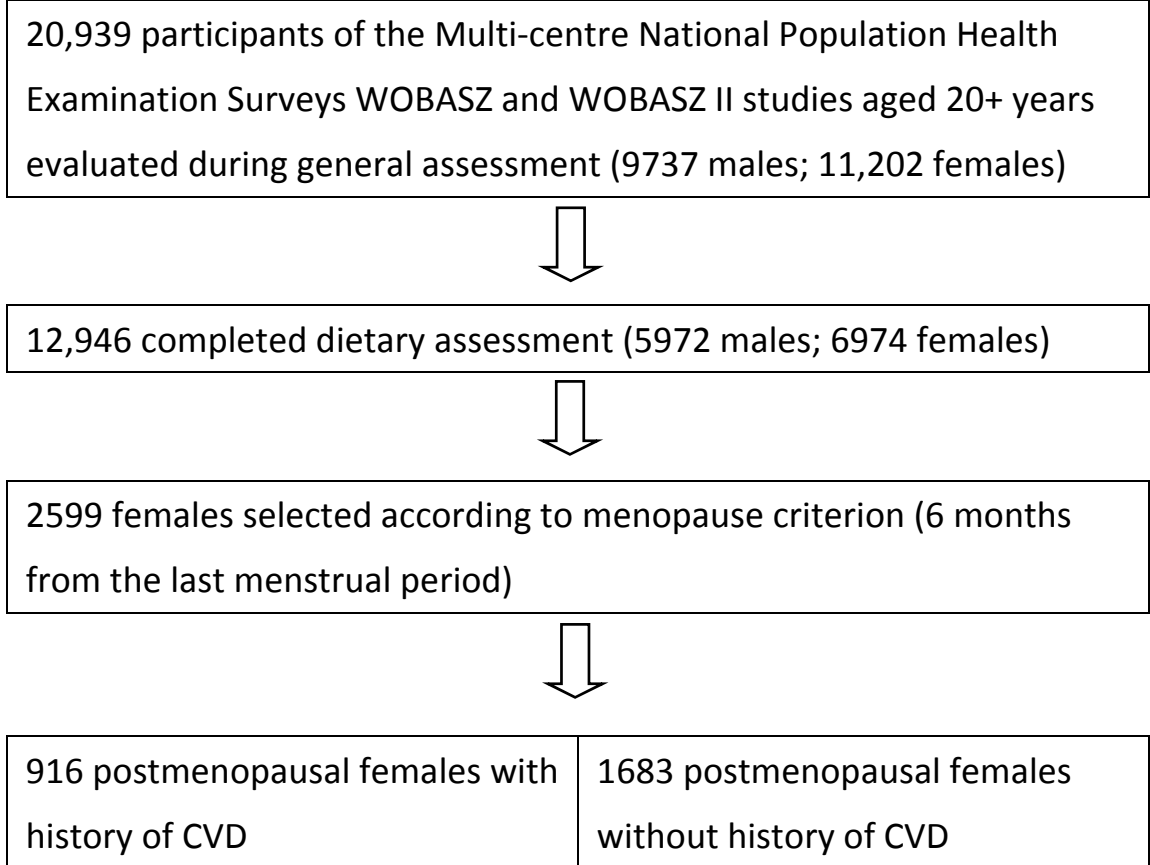

Figure 1. Flow-chart of study participants. CVD: cardiovascular disease.

\subsection{Data Collection}

Data on the level of education (under middle, middle, high), family history of CVD (yes/no), health status (hypertension, myocardial infarction, stroke, diabetes), menopause-hormone therapy (yes/no), leisure-time physical activity (low, middle, high), alcohol intake (pure ethanol g/day) and smoking habit (current smokers, past smokers, never smokers), and the type and dose of medication being taken were collected from the standardized questionnaire designed for the WOBASZ study. Cardiovascular disease classification has been adopted in accordance with the World Health Organization (WHO) [17], where CVDs due to atherosclerosis include ischaemic heart disease or coronary artery disease (e.g., myocardial infarction), cerebrovascular disease (e.g., stroke), diseases of the aorta and arteries, including hypertension and peripheral vascular disease.

Measurements of systolic blood pressure (SBP) and diastolic blood pressure (DBP) were taken three times on the right arm after $5 \mathrm{~min}$ of resting in a sitting position in one-minute intervals and the final SBP and DBP were calculated as an average of the second and third measurements. Hypertension was recognized for SBP $\geq 140 \mathrm{mmHg}$ or $\mathrm{DBP} \geq 90 \mathrm{mmHg}$ or when antihypertensive drugs were used. Hypercholesterolemia was diagnosed if total blood cholesterol was $\geq 5 \mathrm{mmol} / \mathrm{L}$ or LDL-cholesterol levels were $\geq 3 \mathrm{mmol} / \mathrm{L}$ or the participant was taking a lipid-lowering medication. Diabetes was deemed present when blood glucose level was $\geq 7.0 \mathrm{mmol} / \mathrm{L}$ or diabetes was declared in an interview.

Body measurements, such as height, body mass and waist circumference, were taken by the personnel trained in standard procedures. The body mass index (BMI) was calculated from weight in kilograms divided by the square of height in meters. The participants were diagnosed with central obesity, when their waist circumference was $\geq 80 \mathrm{~cm}$, which is a cut off value for European females. Biochemical analyses, i.e., fasting glucose and total cholesterol, were carried out at a single location, Diagnostyka Central Laboratory at the Institute of Cardiology in Warsaw. A general description of the study group stratified by CVD status has been given in Table 1. A more detailed description of the CVD women and those without past history of CVD has been published elsewhere [18]. 
Table 1. Descriptive statistics of the studied postmenopausal women with and without CVD *

\begin{tabular}{|c|c|c|c|c|c|}
\hline \multirow[b]{2}{*}{ Characteristics } & \multicolumn{2}{|c|}{$\begin{array}{l}\text { Women Diagnosed with CVD } \\
\qquad n=916\end{array}$} & \multicolumn{2}{|c|}{$\begin{array}{c}\text { Women without CVD } \\
n=1683\end{array}$} & \multirow[b]{2}{*}{$p$} \\
\hline & Mean \pm SD & $\begin{array}{c}\text { Median } \\
\text { (25-75 Percentile) }\end{array}$ & Mean \pm SD & $\begin{array}{c}\text { Median } \\
\text { (25-75 Percentile) }\end{array}$ & \\
\hline Age (years) & $65.48+9.21$ & $\begin{array}{c}65.00 \\
(58.00-72.00)\end{array}$ & $60.98+8.41$ & $\begin{array}{c}60.00 \\
(55.00-66.00)\end{array}$ & $<0.0001$ \\
\hline Fasting glucose (mmol/L) & $5.7 \pm 1.9$ & $\begin{array}{c}5.14 \\
(4.72-5.80) \\
\end{array}$ & $5.5 \pm 1.6$ & $\begin{array}{c}5.19 \\
(4.72-5.77) \\
\end{array}$ & 0.531 \\
\hline Total cholesterol (mmol/L) & $5.4 \pm 1.2$ & $\begin{array}{c}5.43 \\
(4.64-6.20) \\
\end{array}$ & $5.8 \pm 1.3$ & $\begin{array}{c}5.76 \\
(5.06-6.53) \\
\end{array}$ & $<0.0001$ \\
\hline Energy from food (kcal/day) & $1517 \pm 580$ & $\begin{array}{c}1438 \\
(1130-1831)\end{array}$ & $1653 \pm 628$ & $\begin{array}{c}1587 \\
(1220-1976)\end{array}$ & $<0.0001$ \\
\hline Intake of vegetables (g/day) & $215 \pm 156$ & $\begin{array}{c}189 \\
(104-299) \\
\end{array}$ & $214 \pm 155$ & $\begin{array}{c}187 \\
(100-302) \\
\end{array}$ & 0.850 \\
\hline Intake of fruits (g/day) & $214 \pm 226$ & $\begin{array}{c}150 \\
(25-300)\end{array}$ & $217 \pm 227$ & $\begin{array}{c}150 \\
(10-320)\end{array}$ & 0.989 \\
\hline Intake of tea (g/day) & $361 \pm 252$ & $\begin{array}{c}400 \\
(200-500)\end{array}$ & $348 \pm 251$ & $\begin{array}{c}300 \\
(200-500)\end{array}$ & 0.180 \\
\hline Intake of coffee (g/day) & $130 \pm 166$ & $\begin{array}{c}0 \\
(0-250)\end{array}$ & $175 \pm 172$ & $\begin{array}{c}200 \\
(0-250)\end{array}$ & $<0.0001$ \\
\hline \multirow[t]{2}{*}{$\begin{array}{l}\text { Intake of alcohol } \\
\text { (pure ethanol g/day) }\end{array}$} & $0.69 \pm 2.67$ & $\begin{array}{c}0.15 \\
(0-0.50)\end{array}$ & $0.99 \pm 2.86$ & $\begin{array}{c}0.25 \\
(0-0.80) \\
\end{array}$ & $<0.0001$ \\
\hline & \multicolumn{2}{|r|}{$\%$} & \multicolumn{2}{|r|}{$\%$} & \\
\hline Family history of CVD (\%) & \multicolumn{2}{|r|}{39.4} & \multicolumn{2}{|r|}{36.1} & 0.098 \\
\hline \multicolumn{6}{|l|}{ Diseases (\%) } \\
\hline Hypertension & \multicolumn{2}{|r|}{73.1} & \multicolumn{2}{|r|}{57.3} & $<0.0001$ \\
\hline Myocardial infarction & \multicolumn{2}{|r|}{12.6} & \multicolumn{2}{|r|}{0} & $<0.0001$ \\
\hline Stroke & \multicolumn{2}{|r|}{7.9} & \multicolumn{2}{|r|}{0} & $<0.0001$ \\
\hline Diabetes & \multicolumn{2}{|r|}{19.8} & \multicolumn{2}{|r|}{13.4} & $<0.0001$ \\
\hline Hypercholesterolemia (\%) & \multicolumn{2}{|r|}{79.7} & \multicolumn{2}{|r|}{84.1} & 0.0056 \\
\hline \multicolumn{6}{|l|}{ BMI $\left[\mathrm{kg} / \mathrm{m}^{2}\right](\%)$} \\
\hline Underweight (BMI < 18.5) & \multicolumn{2}{|r|}{0.3} & \multicolumn{2}{|r|}{0.7} & \\
\hline Normal (BMI 18.5-24.99) & \multicolumn{2}{|r|}{20.2} & \multicolumn{2}{|r|}{27.8} & $<0.0001$ \\
\hline Overweight (BMI 25-29.99) & \multicolumn{2}{|r|}{35.2} & \multicolumn{2}{|r|}{39.3} & \\
\hline Obesity (BMI > 30) & & 44.3 & & 32.2 & \\
\hline Central obesity (\%) & & 88.3 & & 82.8 & $<0.0001$ \\
\hline Smoking status (\%) & & & & & \\
\hline Current smokers & & 12.5 & & 20.9 & \\
\hline Past smokers & & 18.6 & & 17.7 & $<0.0001$ \\
\hline Never smokers & & 68.9 & & 61.4 & \\
\hline Leisure-time physical activity (\%) & & & & & \\
\hline Low level & & 52.1 & & 47.8 & \\
\hline Middle level & & 16.5 & & 18.4 & 0.107 \\
\hline High level & & 31.3 & & 33.8 & \\
\hline Level of education (\%) & & & & & \\
\hline Under middle & & 61.0 & & 55.2 & $0 \cap 09$ \\
\hline Middle & & 31.9 & & 35.4 & 0.009 \\
\hline High & & 7.1 & & 9.5 & \\
\hline Menopause hormone therapy (\%) & & 3.8 & & 4.9 & 0.178 \\
\hline
\end{tabular}

\subsection{Dietary Assessment}

Nutritional data were collected by qualified interviewers using a single 24-h dietary recall, which revealed that 367 dishes, food items and beverages consumed by the participants were lignan sources. Individual components of complex dishes were extracted using dish recipes from the Polish Food Composition Tables [19]. These recipes give the amounts of food items required for $100 \mathrm{~g}$ dish portion, with consideration of yield factors. The quantitative composition of plant components of 
ready-to-eat foods was obtained from food labels. For fruit yoghurt, for example, a typical amount of $5 \%$ added fruit was included in the calculation. Data on tea type (black, green, herbal) were not collected. By default, it was assumed that black tea, the most popular tea in Poland, would be consumed.

\subsection{Estimation of Total and Individual Lignan Intakes}

Data on food content of lignans: lariciresinol (LARI), matairesinol (MAT), pinoresinol (PINO) and secoisolariciresinol (SECO) were collected from the available lignan databases [20-22]. The primary source of lignan values in this study was Dutch lignan database [20]. The lignan contents of beverages, nuts, seeds, and oils were taken from Kuhnle et al. [21]. The Thompson et al. [22] database was used when data on lignan content were missing. PINO content in onions, parsnips, fruit jam, apples, LARI content in parsnips and jam, and MAT content in onion, pepper, cherries and cocoa products were not found in literature. Daily intakes of individual lignans were determined by multiplying the daily consumption of individual food items by the respective lignan content in these food items. Individual lignan intakes were summed to obtain total lignan intake.

\subsection{Statistical Analysis}

Descriptive statistics were used for the determination of means, standard deviations (SD), median, percentile and for the percentage analyses of the total and individual lignan dietary intakes.

Odds ratios (ORs) and 95\% confidence intervals (CIs) of CVD incidence, hypertension, hypercholesterolemia and obesity according to the lignan intake were computed, using logistic regression analysis, with adjustment for various potential confounders. Where possible, continuous variables were used for the reason of high sensitivity. The regression models included adjustments for age (continuously), education (under-middle, middle, high), BMI (continuous variable), smoking (current, past, never), leisure-time physical activity (low, middle, high), systolic blood pressure (continuously), energy intake (continuously), alcohol intake (continuously), glucose level (continuously), cholesterol level (continuously).

All tests of statistical significance were two-tailed. SAS version 9.2 (SAS Institute, Inc., Cary, NC, USA) was used for all the statistical analyses.

\section{Results}

In the study group of postmenopausal women, the prevalence of CVD was $35.2 \%$, while the prevalence of hypercholesterolemia $82.5 \%$, hypertension $62.9 \%$ and central obesity $84.7 \%$.

Tables 2-6 illustrate contributions of food categories and individual food products to the intakes of total and individual lignans. The total lignan intake in the CVD women was $1130.7 \mu \mathrm{g} / \mathrm{day}$ and $1095.1 \mu \mathrm{g} /$ day in the non-CVD women $(p=0.925)$. Differences in the intake of secoisolariciresinol, pinoresinol, lariciresinol, matairesinol between the groups of women with and without CVD were not statistically significant.

Percentages of individual lignan intakes were also examined. In the CVD women, secoisolariciresinol accounted for $50.15 \%$ lignan intake from plant foods, as compared to $44.8 \%$ in the control. Pinoresinol, lariciresinol and matairesinol contributed to the total lignan intakes of CVD and non-CVD women in $24.0 \%$ vs. $26.1 \%, 22.7 \%$ vs. $26.1 \%$ and $3.1 \%$ vs. $2.9 \%$, respectively.

Total dietary lignan sources in descending order were: vegetables $>$ nuts and seeds $>$ beverages $>$ fruit $>$ cereals $>$ other food categories $>$ vegetable fats (Table 2). The predominant individual dietary lignan sources for both groups of women included six products: flaxseed, cabbage, tea, potato, legumes, and rye bread for CVD women ( $71 \%$ of the total intake) and flaxseed, cabbage, tea, potato, legumes, broccoli and cauliflower for non-CVD women (64\% of the total lignan intake). The non-CVD women consumed significantly more total lignans from vegetable fats and from foods classified as "other food categories", although their contribution to total lignan intake was negligible. 
Table 2. Contributions of food categories and individual food products to total lignan (LIG) intake in women with and without CVD.

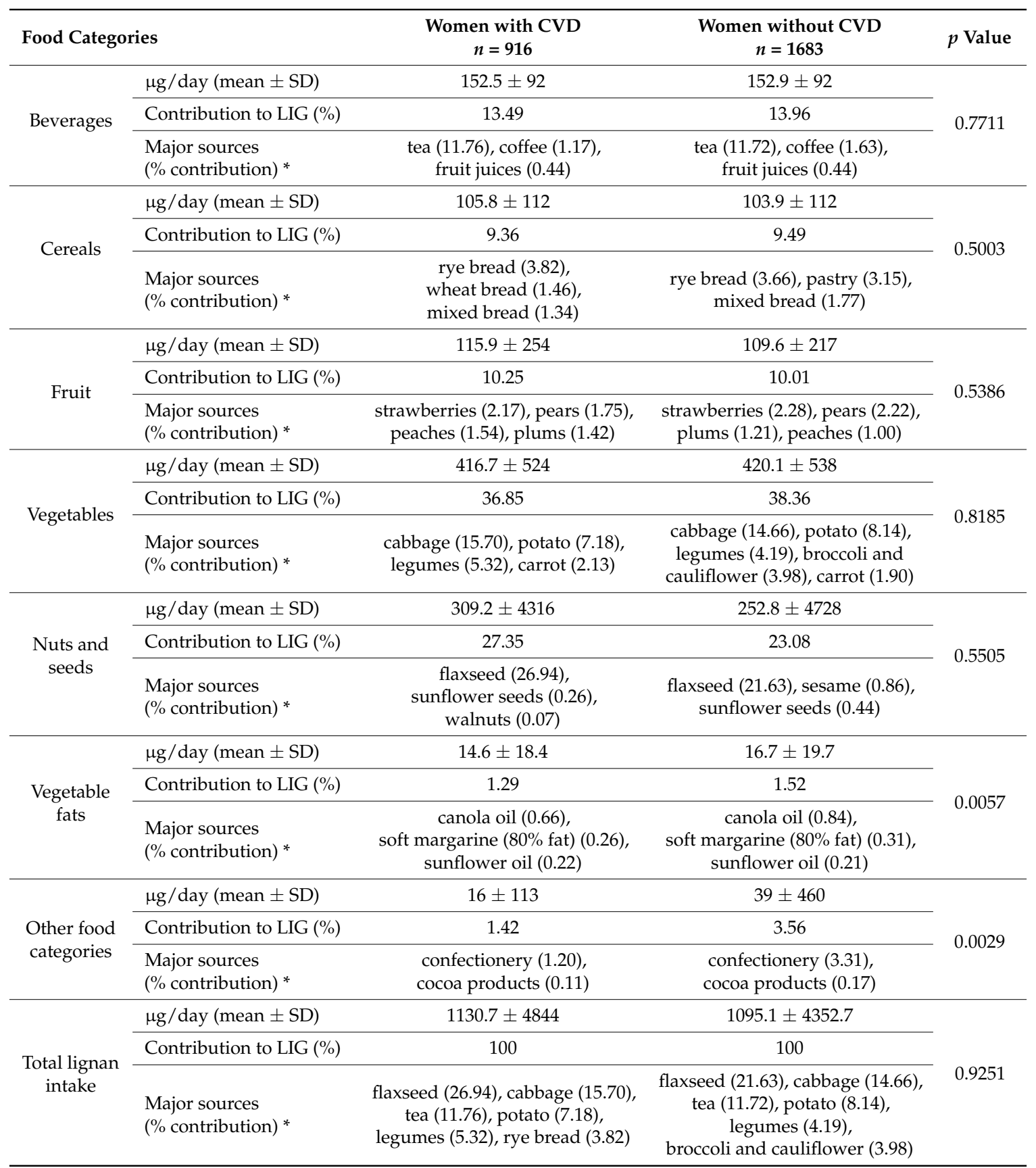

* In the total and each food category, only individual food products with the strongest impact on the total lignan intakes were listed.

The predominant sources of secoisolariciresinol were nuts and seeds in the CVD women (52\%) and in the non-CVD women (47\%) (Table 3). Dietary SECO sources in descending order were: nuts and seeds $>$ beverages $>$ vegetables $>$ cereals $>$ fruit $>$ other food categories $>$ vegetable fats. Flaxseed and tea were among the individual SECO food sources with the highest impact on its consumption in both groups of women (71\% in CVD and $68 \%$ in non-CVD). The CVD-free women consumed significantly more SECO from vegetable fats and from "other food categories", although their contribution to SECO intake was minimum. 
Table 3. Contributions of food categories and individual food products to secoisolariciresinol (SECO) intake in women with and without CVD.

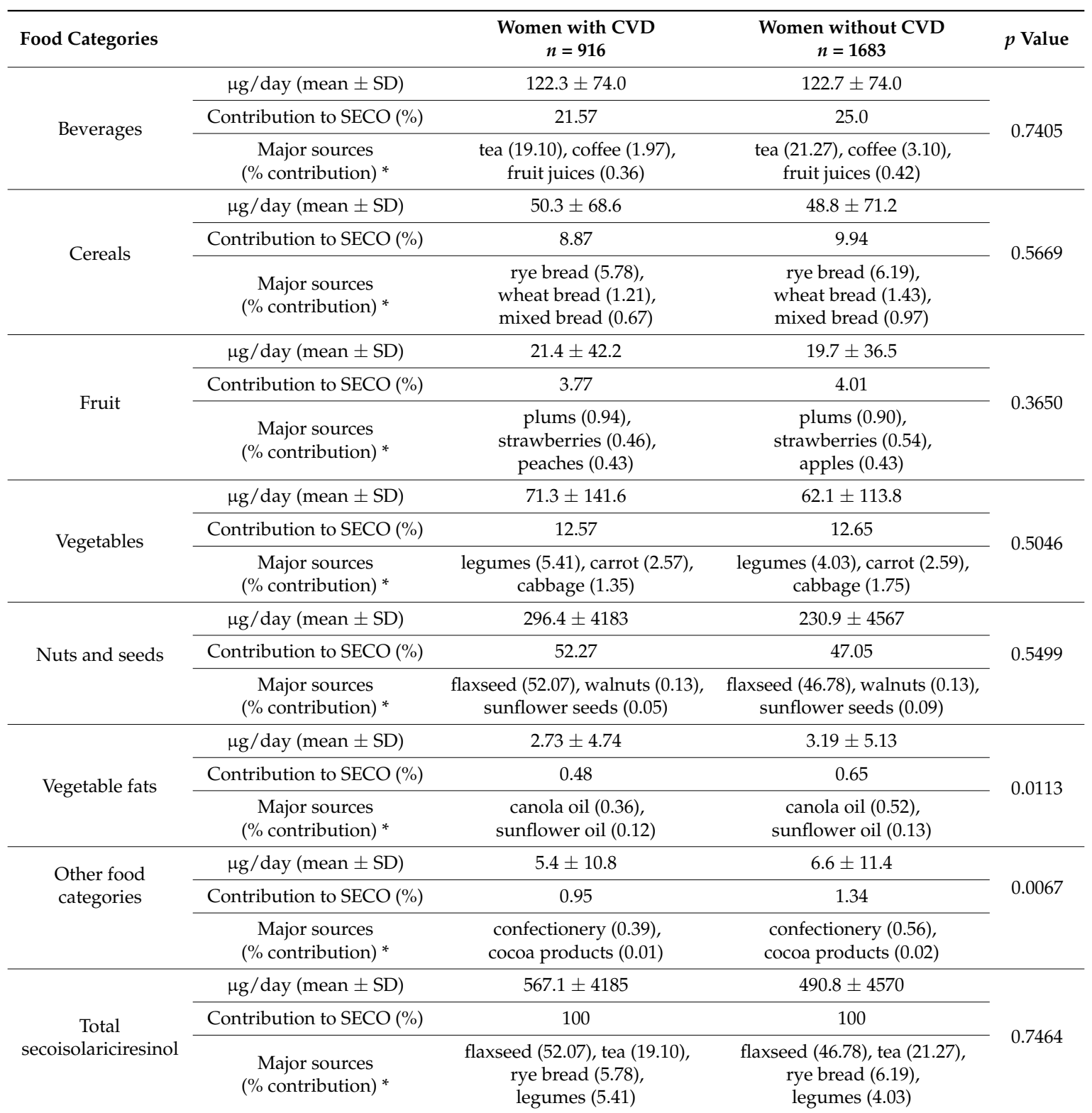

* In the total and each food category, only individual food products with the strongest impact on SECO intakes were listed.

The predominant PINO sources both for the CVD and non-CVD women were vegetables ( $\sim 61 \%$ and $56 \%$, respectively) (Table 4$)$. The dietary PINO sources in descending order were: vegetables $>$ fruit $>$ cereals $>$ other food categories $>$ beverages $>$ vegetable fats $>$ nuts and seeds. Of the PINO sources, cabbage intake was the main contributor ( $43 \%$ in CVD and $36 \%$ in non-CVD women). The non-CVD women consumed significantly more PINO from vegetable fats and from "other food categories". Their contribution to PINO intake was minor. 
Table 4. Contributions of food categories and individual food products to pinoresinol (PINO) intake in women with and without CVD.

\begin{tabular}{|c|c|c|c|c|}
\hline Food Catego & & $\begin{array}{l}\text { Women with CVD } \\
\qquad n=916\end{array}$ & $\begin{array}{l}\text { Women without CVD } \\
\qquad n=1683\end{array}$ & $p$ Value \\
\hline \multirow{3}{*}{ Beverages } & $\mu \mathrm{g} /$ day $($ mean $\pm \mathrm{SD})$ & $16.1 \pm 11.3$ & $15.7 \pm 11.6$ & \multirow{3}{*}{0.5580} \\
\hline & Contribution to PINO (\%) & 5.93 & 5.49 & \\
\hline & $\begin{array}{c}\text { Major sources } \\
(\% \text { contribution }) *\end{array}$ & $\begin{array}{l}\text { tea }(5.30), \text { fruit juices }(0.38) \text {, } \\
\text { beer }(0.12) \text {, coffee }(0.10)\end{array}$ & $\begin{array}{l}\text { tea }(4.86), \text { fruit juices }(0.33) \text {, } \\
\text { beer }(0.13) \text {, coffee }(0.12)\end{array}$ & \\
\hline \multirow{3}{*}{ Cereals } & $\mu \mathrm{g} /$ day $($ mean $\pm \mathrm{SD})$ & $20.4 \pm 20.2$ & $21.7 \pm 24.8$ & \multirow{3}{*}{0.0901} \\
\hline & Contribution to PINO (\%) & 7.51 & 7.59 & \\
\hline & $\begin{array}{c}\text { Major sources } \\
(\% \text { contribution }) *\end{array}$ & $\begin{array}{c}\text { mixed bread (1.96), } \\
\text { wheat bread }(1.41), \\
\text { breakfast cereals }(1.38) \text {, }\end{array}$ & $\begin{array}{c}\text { mixed bread (2.32), } \\
\text { breakfast cereals }(1.58) \text {, } \\
\text { wheat bread }(1.22)\end{array}$ & \\
\hline \multirow{3}{*}{ Fruit } & $\mu \mathrm{g} /$ day $($ mean $\pm \mathrm{SD})$ & $46.7 \pm 129.5$ & $40.7 \pm 104.2$ & \multirow{3}{*}{0.3223} \\
\hline & Contribution to PINO (\%) & 17.20 & 14.23 & \\
\hline & $\begin{array}{c}\text { Major sources } \\
(\% \text { contribution }) *\end{array}$ & $\begin{array}{c}\text { strawberries (4.71), } \\
\text { peaches (3.98), plums (3.26) }\end{array}$ & $\begin{array}{c}\text { strawberries (4.55), plums (2.57), } \\
\text { peaches }(2.40)\end{array}$ & \\
\hline \multirow{3}{*}{ Vegetables } & $\mu \mathrm{g} /$ day $($ mean $\pm \mathrm{SD})$ & $165.6 \pm 312.5$ & $160.7 \pm 304.9$ & \multirow{3}{*}{0.3921} \\
\hline & Contribution to PINO (\%) & 60.99 & 56.19 & \\
\hline & $\begin{array}{c}\text { Major sources } \\
(\% \text { contribution }) *\end{array}$ & $\begin{array}{c}\text { cabbage (43.17), potato }(7.40) \\
\text { legumes }(4.09)\end{array}$ & $\begin{array}{c}\text { cabbage (36.19), potato (7.69), } \\
\text { legumes (3.95) }\end{array}$ & \\
\hline \multirow{3}{*}{$\begin{array}{l}\text { Nuts and } \\
\text { seeds }\end{array}$} & $\mu \mathrm{g} /$ day $($ mean $\pm \mathrm{SD})$ & $3.67 \pm 47.2$ & $10.2 \pm 257.4$ & \multirow{3}{*}{0.5429} \\
\hline & Contribution to PINO (\%) & 1.35 & 3.57 & \\
\hline & $\begin{array}{l}\text { Major sources } \\
(\% \text { contribution })^{*}\end{array}$ & $\begin{array}{c}\text { flaxseed (1.22), } \\
\text { sunflower seeds }(0.11), \\
\text { pumpkin seeds }(0.02)\end{array}$ & $\begin{array}{l}\text { flaxseed }(0.90), \text { sesame }(2.45) \\
\quad \text { sunflower seeds }(0.16)\end{array}$ & \\
\hline \multirow{3}{*}{$\begin{array}{l}\text { Vegetable } \\
\text { fats }\end{array}$} & $\mu \mathrm{g} /$ day $($ mean $\pm S D)$ & $11.3 \pm 13.4$ & $12.9 \pm 14.3$ & \multirow[b]{3}{*}{0.0062} \\
\hline & Contribution to PINO (\%) & 4.16 & 4.51 & \\
\hline & $\begin{array}{c}\text { Major sources } \\
(\% \text { contribution }) *\end{array}$ & $\begin{array}{c}\text { canola oil (1.89), } \\
\text { soft margarine ( } 80 \% \text { fat) }(1.03), \\
\text { sunflower oil }(0.62), \\
\text { soft margarine ( } 60 \% \text { fat) }(0.40)\end{array}$ & $\begin{array}{c}\text { canola oil (2.21), soft margarine } \\
\text { (80\% fat) }(1.13), \\
\text { sunflower oil (0.57), } \\
\text { soft margarine (60\% fat) }(0.33)\end{array}$ & \\
\hline \multirow{3}{*}{$\begin{array}{l}\text { Other food } \\
\text { categories }\end{array}$} & $\mu \mathrm{g} /$ day $($ mean $\pm S D)$ & $19.03 \pm 83.8$ & $37.0 \pm 344.9$ & \multirow{3}{*}{0.0249} \\
\hline & Contribution to PINO (\%) & 7.02 & 12.93 & \\
\hline & $\begin{array}{c}\text { Major sources } \\
(\% \text { contribution }) *\end{array}$ & $\begin{array}{l}\text { confectionery }(2.14), \\
\text { cocoa products }(0.24),\end{array}$ & $\begin{array}{l}\text { confectionery }(7.55), \\
\text { cocoa products }(0.32),\end{array}$ & \\
\hline \multirow{3}{*}{$\begin{array}{c}\text { Total } \\
\text { pinoresinol }\end{array}$} & $\mu \mathrm{g} /$ day $($ mean $\pm S D)$ & $271.5 \pm 356.1$ & $286.0 \pm 548.0$ & \multirow{3}{*}{0.4899} \\
\hline & Contribution to PINO (\%) & 100 & 100 & \\
\hline & & $\begin{array}{c}\text { cabbage (43.17), potato }(7.40) \\
\text { tea }(5.30)\end{array}$ & $\begin{array}{l}\text { cabbage (36.19), potato (7.69), } \\
\text { confectionery }(7.55)\end{array}$ & \\
\hline
\end{tabular}

* In the total and each food category, only individual food products with the strongest impact on PINO intakes were listed.

Vegetables were the main source of LARI in both study groups ( $>65 \%)$ (Table 5). Dietary LARI sources in descending order were: vegetables $>$ fruit $>$ cereals $>$ other food categories $>$ nuts and seeds $>$ beverages $>$ vegetable fats. Individual LARI dietary sources were potato and cabbage in both groups of women, and pear in the CVD women (contribution of 50\% to total lignans), while broccoli and cauliflower in the controls (contribution 48\%). The non-CVD women consumed significantly more LARI from beverages, vegetable fats and from foods classified as "other food categories", although their contribution to LARI intake was negligible. 
Table 5. Contributions of food categories and individual food products to lariciresinol (LARI) intake in women with and without CVD.

\begin{tabular}{|c|c|c|c|c|}
\hline \multicolumn{2}{|c|}{ Food Categories } & $\begin{array}{l}\text { Women with CVD } \\
\qquad n=916\end{array}$ & $\begin{array}{l}\text { Women without CVD } \\
\qquad n=1683\end{array}$ & $p$ Value \\
\hline \multirow{3}{*}{ Beverages } & $\mu \mathrm{g} /$ day $($ mean $\pm \mathrm{SD})$ & $3.45 \pm 5.72$ & $3.86 \pm 6.14$ & \multirow{3}{*}{0.0001} \\
\hline & Contribution to LARI (\%) & 1.35 & 1.35 & \\
\hline & $\begin{array}{c}\text { Major sources } \\
(\% \text { contribution }) *\end{array}$ & $\begin{array}{c}\text { fruit juices }(0.53), \text { coffee }(0.46), \\
\text { tea }(0.28)\end{array}$ & $\begin{array}{c}\text { coffee }(0.55), \text { fruit juices }(0.44), \\
\text { tea }(0.24)\end{array}$ & \\
\hline \multirow{3}{*}{ Cereals } & $\mu \mathrm{g} /$ day $($ mean $\pm \mathrm{SD})$ & $27.6 \pm 44.4$ & $25.9 \pm 36.2$ & \multirow{3}{*}{0.1919} \\
\hline & Contribution to LARI (\%) & 10.78 & 9.06 & \\
\hline & $\begin{array}{c}\text { Major sources } \\
(\% \text { contribution }) *\end{array}$ & $\begin{array}{l}\text { groats and grains (3.85), } \\
\text { mixed bread (3.85) }\end{array}$ & $\begin{array}{l}\text { mixed bread }(3.45) \\
\text { pastry }(3.45)\end{array}$ & \\
\hline \multirow{3}{*}{ Fruit } & $\mu \mathrm{g} /$ day $($ mean $\pm \mathrm{SD})$ & $45.8 \pm 111.4$ & $47.5 \pm 106.6$ & \multirow{3}{*}{0.5872} \\
\hline & Contribution to LARI (\%) & 17.88 & 16.22 & \\
\hline & $\begin{array}{c}\text { Major sources } \\
(\% \text { contribution }) *\end{array}$ & $\begin{array}{c}\text { pear }(7.69), \text { strawberries }(3.85), \\
\text { citrus fruits }(3.85)\end{array}$ & $\begin{array}{c}\text { pear }(6.90), \text { strawberries }(3.45), \\
\text { citrus fruits }(3.45)\end{array}$ & \\
\hline \multirow{3}{*}{ Vegetables } & $\mu \mathrm{g} /$ day $($ mean $\pm \mathrm{SD})$ & $168.1 \pm 170.6$ & $189.5 \pm 241.7$ & \multirow{3}{*}{0.1081} \\
\hline & Contribution to LARI (\%) & 65.64 & 66.31 & \\
\hline & $\begin{array}{c}\text { Major sources } \\
(\% \text { contribution }) *\end{array}$ & $\begin{array}{l}\text { potato (23.08), cabbage (19.23), } \\
\text { broccoli and cauliflower (3.85), } \\
\text { tomato }(3.85), \text { carrot }(3.85)\end{array}$ & $\begin{array}{l}\text { potato }(20.69) \text {, cabbage }(17.24), \\
\text { broccoli and cauliflower }(10.34), \\
\text { tomato }(3.45), \text { carrot }(3.45)\end{array}$ & \\
\hline \multirow[b]{3}{*}{$\begin{array}{l}\text { Nuts and } \\
\text { seeds }\end{array}$} & $\mu \mathrm{g} /$ day $($ mean $\pm \mathrm{SD})$ & $5.39 \pm 58.1$ & $7.60 \pm 105.7$ & \multirow[b]{3}{*}{0.5453} \\
\hline & Contribution to LARI (\%) & 2.10 & 2.66 & \\
\hline & $\begin{array}{c}\text { Major sources } \\
(\% \text { contribution }) *\end{array}$ & $\begin{array}{c}\text { flaxseed }(1.50), \\
\text { sunflower seeds }(0.47), \\
\text { pumpkin seeds }(0.1), \\
\text { walnuts }(0.02)\end{array}$ & $\begin{array}{l}\text { flaxseed (1.05), sesame }(0.76) \\
\text { sunflower seeds }(0.68)\end{array}$ & \\
\hline \multirow{3}{*}{$\begin{array}{l}\text { Vegetable } \\
\text { fats }\end{array}$} & $\mu \mathrm{g} /$ day $($ mean $\pm \mathrm{SD})$ & $0.567 \pm 0.671$ & $0.644 \pm 0.716$ & \multirow{3}{*}{0.0062} \\
\hline & Contribution to LARI (\%) & 0.22 & 0.23 & \\
\hline & $\begin{array}{c}\text { Major sources } \\
(\% \text { contribution }) *\end{array}$ & $\begin{array}{l}\text { canola oil }(0.10), \\
\text { soft margarine }(80 \% \text { fat })(0.05), \\
\text { sunflower oil }(0.03)\end{array}$ & $\begin{array}{l}\text { canola oil }(0.11), \text { soft } \\
\text { margarine }(80 \% \text { fat })(0.06), \\
\text { sunflower oil }(0.03)\end{array}$ & \\
\hline \multirow{3}{*}{$\begin{array}{l}\text { Other food } \\
\text { categories }\end{array}$} & $\mu \mathrm{g} /$ day $($ mean $\pm \mathrm{SD})$ & $5.76 \pm 26.2$ & $11.44 \pm 106.1$ & \multirow{3}{*}{0.0263} \\
\hline & Contribution to LARI (\%) & 2.25 & 4.00 & \\
\hline & $\begin{array}{c}\text { Major sources } \\
(\% \text { contribution }) *\end{array}$ & $\begin{array}{l}\text { confectionery }(1.75) \\
\text { cocoa products }(0.22)\end{array}$ & $\begin{array}{l}\text { confectionery }(3.53) \\
\text { cocoa products }(0.29)\end{array}$ & \\
\hline \multirow{3}{*}{$\begin{array}{l}\text { Total } \\
\text { lariciresinol }\end{array}$} & $\mu \mathrm{g} /$ day $($ mean $\pm \mathrm{SD})$ & $256.1 \pm 222.4$ & $285.8 \pm 320.5$ & \multirow{3}{*}{0.0614} \\
\hline & Contribution to LARI (\%) & 100 & 100 & \\
\hline & $\begin{array}{c}\text { Major sources } \\
(\% \text { contribution }) *\end{array}$ & $\begin{array}{c}\text { potato (23.08), cabbage (19.23), } \\
\text { pear }(7.69)\end{array}$ & $\begin{array}{l}\text { potato }(20.69) \text {, cabbage }(17.24) \text {, } \\
\text { broccoli and cauliflower }(10.34)\end{array}$ & \\
\hline
\end{tabular}

* In the total and each food category, only individual food products with the strongest impact on LARI intakes were listed.

Three food groups were substantial MAT sources: vegetables, beverages and cereals in a range of $21.00-32.62 \%$ for the individual food group (Table 6). They together accounted for $83.22 \%$ of MAT in the CVD and for $79.60 \%$ in the non-CVD women. Dietary MAT sources in descending order were: vegetables $>$ beverages $>$ cereals $>$ nuts and seeds $>$ fruit $>$ other food categories $>$ vegetable fats. Tea, legumes and rye bread were the main individual food sources that contributed to MAT intake in $62.7 \%$ (CVD) and in 55.03\% (non-CVD). The non-CVD women consumed significantly more MAT from vegetable fats and from "other food categories", although their contribution to MAT intake was negligible. 
Table 6. Contributions of food categories and individual food products to matairesinol (MAT) intake in women with and without CVD.

\begin{tabular}{|c|c|c|c|c|}
\hline Food Catego & & $\begin{array}{l}\text { Women with CVD } \\
\qquad n=916\end{array}$ & $\begin{array}{l}\text { Women without CVD } \\
\qquad n=1683\end{array}$ & $p$ Value \\
\hline \multirow{3}{*}{ Beverages } & $\mu \mathrm{g} /$ day $($ mean $\pm \mathrm{SD})$ & $10.7 \pm 6.8$ & $10.6 \pm 6.7$ & \multirow{3}{*}{0.8108} \\
\hline & Contribution to MAT (\%) & 29.72 & 32.62 & \\
\hline & $\begin{array}{l}\text { Major sources } \\
(\% \text { contribution }) *\end{array}$ & $\begin{array}{l}\text { tea (26.56), fruit juices (1.57), } \\
\text { coffee }(1.44)\end{array}$ & $\begin{array}{l}\text { tea }(28.37), \text { coffee }(2.16), \\
\text { fruit juices }(1.70)\end{array}$ & \\
\hline \multirow{3}{*}{ Cereals } & $\mu \mathrm{g} /$ day $($ mean $\pm \mathrm{SD})$ & $7.56 \pm 10.3$ & $7.43 \pm 11.1$ & \multirow{3}{*}{0.3583} \\
\hline & Contribution to MAT (\%) & 21.00 & 22.86 & \\
\hline & $\begin{array}{c}\text { Major sources } \\
(\% \text { contribution }) *\end{array}$ & $\begin{array}{c}\text { rye bread (13.06), } \\
\text { wheat bread }(2.89), \\
\text { breakfast cereals }(2.63)\end{array}$ & $\begin{array}{c}\text { rye bread (13.42), } \\
\text { wheat bread }(3.26), \\
\text { breakfast cereals }(3.00)\end{array}$ & \\
\hline \multirow{3}{*}{ Fruit } & $\mu \mathrm{g} /$ day $($ mean $\pm \mathrm{SD})$ & $1.92 \pm 7.9$ & $1.69 \pm 5.7$ & \multirow{3}{*}{0.2993} \\
\hline & Contribution to MAT ( $\%)$ & 5.33 & 5.20 & \\
\hline & $\begin{array}{c}\text { Major sources } \\
(\% \text { contribution }) *\end{array}$ & $\begin{array}{l}\text { grapes (1.93), } \\
\text { citrus fruits (1.44), } \\
\text { dried fruits (1.20) }\end{array}$ & $\begin{array}{l}\text { citrus fruits (1.80), } \\
\text { grapes }(1.74) \text {, dried fruits }(0.8)\end{array}$ & \\
\hline \multirow{3}{*}{ Vegetables } & $\mu \mathrm{g} /$ day $($ mean $\pm \mathrm{SD})$ & $11.7 \pm 184.6$ & $7.84 \pm 99.9$ & \multirow{3}{*}{0.7974} \\
\hline & Contribution to MAT ( $\%)$ & 32.50 & 24.12 & \\
\hline & $\begin{array}{c}\text { Major sources } \\
(\% \text { contribution }) *\end{array}$ & $\begin{array}{c}\text { legumes (23.03), potato (4.28), } \\
\text { parsnips }(2.72)\end{array}$ & $\begin{array}{c}\text { legumes }(13.60), \text { potato }(5.20), \\
\text { parsnips }(2.74)\end{array}$ & \\
\hline \multirow[b]{3}{*}{$\begin{array}{l}\text { Nuts and } \\
\text { seeds }\end{array}$} & $\mu \mathrm{g} /$ day $($ mean $\pm S D)$ & $3.69 \pm 37.2$ & $4.12 \pm 45.5$ & \multirow[b]{3}{*}{0.5510} \\
\hline & Contribution to MAT (\%) & 10.25 & 12.68 & \\
\hline & $\begin{array}{l}\text { Major sources } \\
(\% \text { contribution }) *\end{array}$ & $\begin{array}{c}\text { flaxseed (6.11), } \\
\text { sunflower seeds }(3.36), \\
\text { pumpkin seeds }(0.71), \\
\text { walnuts }(0.06)\end{array}$ & $\begin{array}{l}\text { sunflower seeds (5.97), } \\
\text { flaxseed (5.26), } \\
\text { pumpkin seed }(0.86), \\
\text { sesame }(0.52)\end{array}$ & \\
\hline \multirow{3}{*}{$\begin{array}{l}\text { Vegetable } \\
\text { fats }\end{array}$} & $\mu \mathrm{g} /$ day $($ mean $\pm \mathrm{SD})$ & $0.0085 \pm 0.010$ & $0.0098 \pm 0.011$ & \multirow{3}{*}{0.0054} \\
\hline & Contribution to MAT (\%) & 0.02 & 0.03 & \\
\hline & $\begin{array}{c}\text { Major sources } \\
(\% \text { contribution }) *\end{array}$ & $\begin{array}{c}\text { canola oil (0.01), } \\
\text { soft margarine ( } 80 \% \text { fat) }(0.01)\end{array}$ & $\begin{array}{c}\text { canola oil (0.01), soft } \\
\text { margarine ( } 80 \% \text { fat) }(0.01)\end{array}$ & \\
\hline \multirow{3}{*}{$\begin{array}{l}\text { Other food } \\
\text { categories }\end{array}$} & $\mu \mathrm{g} /$ day $($ mean $\pm S D)$ & $0.43 \pm 2.10$ & $0.82 \pm 8.26$ & \multirow{3}{*}{0.0033} \\
\hline & Contribution to MAT (\%) & 1.20 & 2.52 & \\
\hline & $\begin{array}{c}\text { Major sources } \\
(\% \text { contribution }) *\end{array}$ & confectionery & confectionery & \\
\hline \multirow{3}{*}{$\begin{array}{l}\text { Total } \\
\text { matairesinol }\end{array}$} & $\mu \mathrm{g} /$ day $($ mean $\pm \mathrm{SD})$ & $36.0 \pm 190$ & $32.5 \pm 111$ & \multirow{3}{*}{0.5174} \\
\hline & Contribution to MAT (\%) & 100 & 100 & \\
\hline & $\begin{array}{c}\text { Major sources } \\
(\% \text { contribution }) *\end{array}$ & $\begin{array}{l}\text { tea }(26.56) \text {, legumes }(23.03), \\
\text { rye bread }(13.06)\end{array}$ & $\begin{array}{l}\text { tea }(28.37) \text {, legumes }(13.60), \\
\text { rye bread }(13.06)\end{array}$ & \\
\hline
\end{tabular}

* In the total and each food category, only individual food products with the strongest impact on MAT intakes were listed.

According to the previous study involving the same participants, women with CVD differed from those without past history of CVD in age, education, smoking habit, cholesterol level, alcohol intake, coffee intake, energy intake, BMI, prevalence of central obesity and hypercholesterolemia ([18] and Table 1). They did not differ in the use of menopause hormone therapy (MHT), leisure-time activity, family history of CVD, consumption of vegetables, fruit and tea, in dietary polyphenol intake (DPI) or dietary antioxidant capacity (DTAC) ([18] and Table 1).

In the multiple adjustment models, total lignans and individual lignans such as pinoresinol, matairesinol and secoisolariciresinol were not associated with the prevalence of CVD, hypercholesterolemia, hypertension and central obesity in the study participants (Table 7). 
Table 7. Association between total and individual lignan intake and prevalence of CVD, hypercholesterolemia, hypertension and central obesity; multivariable analysis.

\begin{tabular}{|c|c|c|c|c|c|}
\hline Variables & & $\begin{array}{c}\text { CVD }^{1} \\
\text { OR }(95 \% \mathrm{CI})\end{array}$ & $\begin{array}{c}\text { Hypercholeste- } \\
\text { Rolemia }^{2} \\
\text { OR }(95 \% \text { CI })\end{array}$ & $\begin{array}{c}\text { Hypertension } \\
\text { OR }(95 \% \text { CI })\end{array}$ & $\begin{array}{c}\text { Central } \\
\text { Obesity } \\
\text { OR }(95 \% \text { CI })\end{array}$ \\
\hline \multirow[t]{2}{*}{$\begin{array}{l}\text { Total lignans } \\
(\mu \mathrm{g} / \text { day })\end{array}$} & Model 1 & $\begin{array}{c}1.002 \\
(0.984 ; 1.020) \\
p=0.8380\end{array}$ & $\begin{array}{c}0.989 \\
(0.972 ; 1.006) \\
p=0.2117\end{array}$ & $\begin{array}{c}0.991 \\
(0.971 ; 1.011) \\
p=0.3522\end{array}$ & $\begin{array}{c}0.981 \\
(0.960 ; 1.003) \\
p=0.0927\end{array}$ \\
\hline & Model 2 & $\begin{array}{c}1.003 \\
(0.986 ; 1.021) \\
p=0.7594\end{array}$ & $\begin{array}{c}0.989 \\
(0.972 ; 1.007) \\
p=0.2277\end{array}$ & $\begin{array}{c}0.991 \\
(0.972 ; 1.011) \\
p=0.3923\end{array}$ & $\begin{array}{c}0.982 \\
(0.961 ; 1.003) \\
p=0.0975\end{array}$ \\
\hline \multirow[t]{2}{*}{$\begin{array}{l}\text { Secoisolariciresinol } \\
\quad(\mu \mathrm{g} / \text { day })\end{array}$} & Model 1 & $\begin{array}{c}1.003 \\
(0.985 ; 1.022) \\
0.7145\end{array}$ & $\begin{array}{c}0.991 \\
(0.972 ; 1.009) \\
p=0.3277\end{array}$ & $\begin{array}{c}0.993 \\
(0.973 ; 1.013) \\
p=0.4939\end{array}$ & $\begin{array}{c}0.978 \\
(0.954 ; 1.002) \\
p=0.0739\end{array}$ \\
\hline & Model 2 & $\begin{array}{c}1.003 \\
(0.985 ; 1.022) \\
0.7136\end{array}$ & $\begin{array}{c}0.991 \\
(0.972 ; 1.009) \\
p=0.3229\end{array}$ & $\begin{array}{c}0.993 \\
(0.973 ; 1.013) \\
p=0.5003\end{array}$ & $\begin{array}{c}0.977 \\
(0.953 ; 1.002) \\
p=0.0742\end{array}$ \\
\hline \multirow[t]{2}{*}{ Pinoresinol ( $\mu \mathrm{g} /$ day) } & Model 1 & $\begin{array}{c}0.944 \\
(0.782 ; 1.139) \\
p=0.5457\end{array}$ & $\begin{array}{c}0.836 \\
(0.695 ; 1.005) \\
p=0.0565\end{array}$ & $\begin{array}{c}0.842 \\
(0.697 ; 1.016) \\
p=0.0734\end{array}$ & $\begin{array}{c}1.126 \\
(0.881 ; 1.455) \\
p=0.3665\end{array}$ \\
\hline & Model 2 & $\begin{array}{c}0.987 \\
(0.821 ; 1.187) \\
p=0.8927\end{array}$ & $\begin{array}{c}0.853 \\
(0.708 ; 1.028) \\
p=0.0958\end{array}$ & $\begin{array}{c}0.868 \\
(0.720 ; 1.047) \\
p=0.1382\end{array}$ & $\begin{array}{c}1.160 \\
(0.886 ; 1.520) \\
p=0.2807\end{array}$ \\
\hline \multirow[t]{2}{*}{$\begin{array}{l}\text { Lariciresinol } \\
(\mu \mathrm{g} / \mathrm{day})\end{array}$} & Model 1 & $\begin{array}{c}0.717 \\
(0.507 ; 1.014) \\
p=0.0597\end{array}$ & $\begin{array}{c}0.656 \\
(0.468 ; 0.921) \\
p=0.0148\end{array}$ & $\begin{array}{c}0.694 \\
(0.505 ; 0.952) \\
p=0.0236\end{array}$ & $\begin{array}{c}1.048 \\
(0.713 ; 1.539) \\
p=0.8128\end{array}$ \\
\hline & Model 2 & $\begin{array}{c}0.815 \\
(0.575 ; 1.154) \\
p=0.2487\end{array}$ & $\begin{array}{c}0.693 \\
(0.488 ; 0.985) \\
p=0.0410\end{array}$ & $\begin{array}{c}0.744 \\
(0.539 ; 1.029) \\
p=0.0736\end{array}$ & $\begin{array}{c}1.109 \\
(0.741 ; 1.659) \\
p=0.6146\end{array}$ \\
\hline \multirow[t]{2}{*}{$\begin{array}{l}\text { Matairesinol } \\
(\mu \mathrm{g} / \text { day })\end{array}$} & Model 1 & $\begin{array}{c}1.347 \\
(0.776 ; 2.338) \\
p=0.2902\end{array}$ & $\begin{array}{c}1.082 \\
(0.459 ; 2.552) \\
p=0.8576\end{array}$ & $\begin{array}{c}1.290 \\
(0.692 ; 2.405) \\
p=0.4228\end{array}$ & $\begin{array}{c}1.128 \\
(0.451 ; 2.817) \\
p=0.7968\end{array}$ \\
\hline & Model 2 & $\begin{array}{c}1.415 \\
(0.811 ; 2.469) \\
p=0.2214\end{array}$ & $\begin{array}{c}1.133 \\
(0.463 ; 2.777) \\
p=0.7842\end{array}$ & $\begin{array}{c}1.347 \\
(0.708 ; 2.564) \\
p=0.3645\end{array}$ & $\begin{array}{c}1.166 \\
(0.455 ; 2.989) \\
p=0.7489\end{array}$ \\
\hline
\end{tabular}

OR: odds ratio; ${ }^{1}$ CVD: Model 1—adjusted for age, smoking, BMI, alcohol intake, education, leisure-time physical activity, SBP, glucose level, cholesterol level, menopause hormone therapy. Model 2-additionally adjusted for energy intake. ${ }^{2}$ Hypercholesterolemia: Model 1-adjusted for age, smoking, BMI, alcohol intake, education, leisure-time physical activity, SBP, glucose level, menopause hormone therapy. Model 2-additionally adjusted for energy intake. ${ }^{3}$ Hypertension: Model 1 -adjusted for age, smoking, BMI, alcohol intake, education, leisure-time physical activity, glucose level, cholesterol level, menopause hormone therapy. Model 2-additionally adjusted for energy intake. ${ }^{4}$ Central obesity: Model 1 -adjusted for age, smoking, alcohol intake, education, leisure-time physical activity, SBP, glucose level, cholesterol level, menopause hormone therapy. Model 2-additionally adjusted for energy intake.

Of the variables tested in this study, the intake of lariciresinol was associated with the reduced odds of hypercholesterolemia (OR 0.656; 95\% CI 0.468-0.921) and hypertension (OR 0.694; 0.505-0.952) (Table 7) in the models adjusted for potential confounders excluding energy intake. When energy intake was added as an adjustment, only OR for lariciresinol and hypercholesterolemia, but not for hypertension, remained statistically significant.

\section{Discussion}

In this group of postmenopausal women, dietary intake of total lignans, as well as the respective lignan types, were not associated with the prevalence of CVD. Recently, we showed an association of the dietary intake of total polyphenols in this group of postmenopausal women with lower prevalence of CVD, but it was not found for the total dietary antioxidant intake [18]. Polyphenols are 
a large group of phytochemicals that share common structural features of phenolic units. The most prevalent groups of dietary polyphenols are phenolic acids and flavonoids, which are responsible for $97 \%$ dietary polyphenol intake, while stilbenes, lignans and other phenolics comprise only the remaining 3\% [1]. Thus, the question arises whether and what role lignans can play for human health. Some observational studies suggest that lignans and their derivatives produced by the intestinal bacteria, called enterolignans, provide protection in some types of cancer, diabetes and in cardiovascular disease [23-28]. Conversely, this finding was not confirmed by several other studies [10,29-32]. Lignans may play potentially beneficial roles associated with aging. In middle age, increased lignan intakes were associated with a less cognitive decline [33]. The other roles of dietary lignans remain largely unknown.

The chemical structure of lignans is similar to that of endogenous estrogens. Lignans are considered the major group of phytoestrogens for Western populations [34-36], although the intakes of phytoestrogens in these populations are low [36]. Our results $1.131 \mathrm{mg} /$ day and $1.095 \mathrm{mg} /$ day fall within the range of lignan intakes in European females. Total lignan intakes by women in five European countries (Denmark, Finland, Italy, Sweden, United Kingdom) calculated from the intakes of four lignans SECO, LARI, PINO, MAT vary from $1.036 \mathrm{mg} /$ day in Finland to $1.563 \mathrm{mg} /$ day in Sweden [2], with similarity of age range in our study to the age range of 45-79 years in the Swedish Mammography Cohort (SMC) [37]. However, contrary to the SMC, which used food frequency questionnaires as dietary assessment methods, ours and other studies applied dietary records or dietary recalls.

To calculate lignan intakes in this study, we used the Dutch lignan database developed by Milder et al. [20], which was used in several other studies [2,10]. Data gaps were supplemented with lignan values from Kuhnle et al. [21] and Thompson et al. [22]. This approach allowed for the addition of data on lignan contents for most of the food products. Dietary patterns of lignan consumption may differ among countries. We found that the general sources of dietary lignans for Polish postmenopausal women (75-77\% intake), considering the amounts that have been ingested, are vegetables (37-38\% total dietary intake), nuts and seeds (23-27\%) as well as beverages (13-14\%), mostly tea. However, in Dutch men and women aged 19-97 years, despite the similarity of primary lignan sources regardless of gender, the lignan sequence was different. Beverages were the first, followed by vegetables, nuts and seeds [38]. In contrast, the main sources of lignans in five European countries according to the Dutch database included cereals and grain products, vegetables, fruit, berries and beverages [2]. In an Italian study which enrolled men and women with a median age of $60 \mathrm{yrs}$, one third of lignan intake came from wine (mostly red), followed by fruits and vegetables [13].

The main dietary lignan source for postmenopausal women in Poland is flaxseed. This fact can be associated with its laxative properties, and flaxseed is frequently used by the elderly to prevent constipation. Linseeds are also present in bread and breakfast products, as part of the healthy food trend. Flax is the most abundant source of lignans out of other food products. Its content of $335 \mathrm{mg} / 100 \mathrm{~g}$ exceeds almost 200 times that of rye seeds [39].

Among the lignans studied, we found that secoisolariciresinol was the main dietary lignan for postmenopausal Polish women. It contributed 50\% to the total lignan intake in the CVD women and $45 \%$ in the non-CVD women, as compared to pinoresinol in $24 \%$ vs. $26 \%$, lariciresinol $23 \%$ vs. $26 \%$, respectively, and matairesinol (3\%) in both groups of the women. These findings are in accordance with the individual lignan intakes by middle aged/elderly men and postmenopausal women in Northern Italy, where secoisolariciresinol accounted for $52 \%$, pinoresinol $17 \%$, lariciresinol $27 \%$, and matairesinol $3 \%$ of total lignans [13]. However, in Dutch men and women, lariciresinol and pinoresinol contributed to $75 \%$ of lignan intake, whereas secoisolariciresinol and matairesinol only for $25 \%$ [38]. In our research, the share of pinoresinol and lariciresinol together was no more than $47-52 \%$. The main lignan intake was from secoisolariciresinol, as mentioned above.

The number of studies that have reported on the associations between individual lignan intakes and heart health is limited. Two studies mention matairesinol. The Zutphen Elderly Study, carried out for over 15 years in Dutch elderly men, revealed that the matairesinol intake was inversely associated 
with the coronary heart disease mortality and cardiovascular disease mortality [10]. On the other hand, in a cross-sectional Italian study, higher matairesinol intake in middle aged/elderly men and postmenopausal women was associated with increased flow mediated dilation [13]. The above studies failed to find significant results for other lignans $[10,13]$. A recent study showed an inverse relationship between pinoresinol and the prevalence of hypertension in Mediterranean adults [40]. For the first time, our findings suggest that lariciresinol can be protective against hypercholestrolemia in postmenopausal women. Until now, lariciresinol has never been mentioned in the literature in the context of altered lipid levels. However, lariciresinol and matairesinol have been found to be protective against hormone-dependent cancers, such as the ovarian and endometrial ones [23].

Our research shows that the most significant food sources of LARI for Polish postmenopausal women are vegetables (more than 65\% in both groups of women), and particularly potato and cabbage. Broccoli and cauliflower are the source of lariciresinol for CVD-free women in contrast to those with CVD. Potatoes are potassium-rich [41], and cruciferous vegetables (Brassica vegetables as cabbages, cauliflower, broccoli) are good sources of vitamin C and phytochemicals (e.g., sulphoraphane), which are essential for CVD prevention [42]. It is likely that some bioactive components present in food, and not only lariciresinol, might account for the cardioprotective effects of these foods. Interestingly, in a prospective study with a mean follow-up of 6.9 years, the consumption of broccoli was associated with insignificant 25-30\% reductions in CVD risk in American women [43].

This observational study has strengths and limitations. The strength was the large sample of postmenopausal participants combined from the WOBASZ and WOBASZ II studies. The study groups of CVD and CVD-free women differed by age, education, serum cholesterol levels, smoking habit, BMI and the intakes of alcohol and energy. These differences were taken into account by using the multiple adjustment models. The advantage of this study was the use of several lignan databases, which allowed elimination of most of data gaps.

The main limitation stemmed from the cross-sectional design which does not address the problem of causality. Another limitation was the use of 24-h dietary recalls that may not reflect long-term food consumption, and a validated food frequency questionnaire would be more powerful than a single 24-h dietary recall. Moreover, this study might have underestimated those lignans which were not present in the available lignan databases. Only four lignans were taken into consideration, although other lignans, such as syringaresinol or sesamin can be metabolized to enterolignans. Finally, the overall response rate in the WOBASZ II study was lower than that in the first WOBASZ study (45.5\% vs. 76\%), which might have had an impact on the study group representativeness.

\section{Conclusions}

This study reinforces the existing concept that the dietary intake of total lignans is not associated with the prevalence of CVD, and provides further evidence that it is not linked to CVD risk factors such as hypertension, hypercholesterolemia and central obesity. However, the intake of the individual lignan, lariciresinol, should be taken into consideration in further studies with regard to its potentially beneficial effect in hypercholesterolemia.

Author Contributions: Conceptualization, A.M.W. and A.W.; Methodology, A.M.W., A.W. and M.E.Z.; Formal Analysis, A.M.W., A.W. and D.S.; Investigation, A.M.W. and A.W.; Resources, A.W., D.S., U.S., A.P. and W.D.; Data Curation, A.W. and D.S.; Writing-Original Draft Preparation, A.M.W.; Writing-Review \& Editing, A.W., M.E.Z., U.S., A.P. and W.D.; Funding Acquisition, A.M.W. and A.W.; A.M.W. and A.W. contributed equally to this work.

Funding: This study was supported by the Cardinal Stefan Wyszynski Institute of Cardiology [Grant No. 2.17/I/16] and by the Medical University of Bialystok [Grant No. N/ST/ZB/16/001/3317].

Acknowledgments: The authors are particularly grateful to the research team of the WOBASZ and WOBASZ II studies for sharing of the data, the collaborators from the field centers for the data collection and to the participants for taking part in the studies.

Conflicts of Interest: The authors declare no conflict of interest. 


\section{References}

1. Witkowska, A.M.; Zujko, M.E.; Waśkiewicz, A.; Terlikowska, K.M.; Piotrowski, W. Comparison of various databases for estimation of dietary polyphenol intake in the population of Polish adults. Nutrients 2015, 7, 9299-9308. [CrossRef] [PubMed]

2. Tetens, I.; Turrini, A.; Tapanainen, H.; Christensen, T.; Lampe, J.W.; Fagt, S.; Håkansson, N.; Lundquist, A.; Hallund, J.; Valsta, L.M.; et al. Phytohealth WP1 working group. Dietary intake and main sources of plant lignans in five European countries. Food Nutr. Res. 2013, 57. [CrossRef] [PubMed]

3. Prasad, K. Antioxidant activity of secoisolariciresinol diglucoside-derived metabolites, secoisolariciresinol, enterodiol, and enterolactone. Int. J. Angiol. 2000, 9, 220-225. [CrossRef] [PubMed]

4. Zhu, Y.; Kawaguchi, K.; Kiyama, R. Differential and directional estrogenic signaling pathways induced by enterolignans and their precursors. PLoS ONE 2017, 12, e0171390. [CrossRef] [PubMed]

5. Corrêa, R.C.G.; Peralta, R.M.; Haminiuk, C.W.I.; Maciel, G.M.; Bracht, A.; Ferreira, I.C.F.R. New phytochemicals as potential human anti-aging compounds: Reality, promise, and challenges. Crit. Rev. Food Sci. Nutr. 2018, 58, 942-957. [CrossRef] [PubMed]

6. De Kat, A.C.; Dam, V.; Onland-Moret, N.C.; Eijkemans, M.J.; Broekmans, F.J.; van der Schouw, Y.T. Unraveling the associations of age and menopause with cardiovascular risk factors in a large population-based study. BMC Med. 2017, 15, 2. [CrossRef]

7. Felmlee, M.A.; Woo, G.; Simko, E.; Krol, E.S.; Muir, A.D.; Alcorn, J. Effects of the flaxseed lignans secoisolariciresinol diglucoside and its aglycone on serum and hepatic lipids in hyperlipidaemic rats. Br. J. Nutr. 2009, 102, 361-369. [CrossRef] [PubMed]

8. Miranda, A.M.; Steluti, J.; Fisberg, R.M.; Marchioni, D.M. Association between polyphenol intake and hypertension in adults and older adults: A population-based study in Brazil. PLoS ONE 2016, 11, e0165791. [CrossRef] [PubMed]

9. Tresserra-Rimbau, A.; Rimm, E.B.; Medina-Remón, A.; Martínez-González, M.A.; de la Torre, R.; Corella, D.; Salas-Salvadó, J.; Gómez-Gracia, E.; Lapetra, J.; Arós, F.; et al. Inverse association between habitual polyphenol intake and incidence of cardiovascular events in the PREDIMED study. Nutr. Metab. Cardiovasc. Dis. 2014, 24, 639-647. [CrossRef] [PubMed]

10. Milder, I.E.; Feskens, E.J.; Arts, I.C.; Bueno-de-Mesquita, H.B.; Hollman, P.C.; Kromhout, D. Intakes of 4 dietary lignans and cause-specific and all-cause mortality in the Zutphen Elderly Study. Am. J. Clin. Nutr. 2006, 84, 400-405. [PubMed]

11. Van der Schouw, Y.T.; Kreijkamp-Kaspers, S.; Peeters, P.H.; Keinan-Boker, L.; Rimm, E.B.; Grobbee, D.E. Prospective study on usual dietary phytoestrogen intake and cardiovascular disease risk in Western women. Circulation 2005, 111, 465-471. [CrossRef] [PubMed]

12. De Kleijn, M.J.; van der Schouw, Y.T.; Wilson, P.W.; Grobbee, D.E.; Jacques, P.F. Dietary intake of phytoestrogens is associated with a favorable metabolic cardiovascular risk profile in postmenopausal U.S. women: The Framingham study. J. Nutr. 2002, 132, 276-282. [CrossRef] [PubMed]

13. Pellegrini, N.; Valtueña, S.; Ardigò, D.; Brighenti, F.; Franzini, L.; Del Rio, D.; Scazzina, F.; Piatti, P.M.; Zavaroni, I. Intake of the plant lignans matairesinol, secoisolariciresinol, pinoresinol, and lariciresinol in relation to vascular inflammation and endothelial dysfunction in middle age-elderly men and post-menopausal women living in Northern Italy. Nutr. Metab. Cardiovasc. Dis. 2010, 20, 64-71. [CrossRef] [PubMed]

14. Broda, G.; Rywik, S. Multicenter national Polish population health status tests-WOBASZ project with defined problems and treatment goals. Kardiol. Pol. 2005, 63 (Suppl. 4), 601-604. (In Polish)

15. Rywik, S.; Kupść, W.; Piotrowski, W.; Broda, G.; Piwoński, J.; Kurjata, P.; Waśkiewicz, A.; Gaździk, D. Multicenter national Polish population health status tests-WOBASZ project. Establishment of methods and logistics. Kardiol. Pol. 2005, 63 (Suppl. 4), 605-613. (In Polish)

16. Drygas, W.; Niklas, A.A.; Piwońska, A.; Piotrowski, W.; Flotyńska, A.; Kwaśniewska, M.; Nadrowski, P.; Puch-Walczak, A.; Szafraniec, K.; Bielecki, W.; et al. Multi-center National Population Health Examination Survey (WOBASZ II study): Assumptions, methods and implementation. Kardiol. Pol. 2015. [CrossRef]

17. Mendis, S.; Puska, P.; Norrving, B. (Eds.) Global Atlas on Cardiovascular Disease Prevention and Control, 1st ed.; World Health Organization in Collaboration with the World Heart Federation; The World Stroke Organization: Geneva, Switzerland, 2011; pp. 2-7. 
18. Witkowska, A.M.; Waśkiewicz, A.; Zujko, M.E.; Szcześniewska, D.; Pająk, A.; Stepaniak, U.; Drygas, W. Dietary polyphenol intake, but not the dietary total antioxidant capacity, is inversely related to cardiovascular disease in postmenopausal Polish women: Results of WOBASZ and WOBASZ II Studies. Oxid. Med. Cell. Longev. 2017, 2017, 5982809. [CrossRef] [PubMed]

19. Kunachowicz, H.; Nadolna, I.; Przygoda, B.; Iwanow, K. Food Composition Tables; PZWL: Warsaw, Poland, 2005.

20. Milder, I.E.; Arts, I.C.; van de Putte, B.; Venema, D.P.; Hollman, P.C. Lignan contents of Dutch plant foods: A database including lariciresinol, pinoresinol, secoisolariciresinol and matairesinol. Br. J. Nutr. 2005, 93, 393-402. [CrossRef] [PubMed]

21. Kuhnle, G.G.C.; Dell'Aquila, C.; Aspinall, S.M.; Runswick, S.A.; Mulligan, A.A.; Bingham, S.A. Phytoestrogen content of beverages, nuts, seeds, and oils. J. Agric. Food Chem. 2008, 56, 7311-7315. [CrossRef] [PubMed]

22. Thompson, L.U.; Boucher, B.A.; Liu, Z.; Cotterchio, M.; Kreiger, N. Phytoestrogen content of foods consumed in Canada, including isoflavones, lignans, and coumestan. Nutr. Cancer 2006, 54, 184-201. [CrossRef] [PubMed]

23. Neill, A.S.; Ibiebele, T.I.; Lahmann, P.H.; Hughes, M.C.; Nagle, C.M.; Webb, P.M.; Australian Ovarian Cancer Study Group; Australian National Endometrial Cancer Study Group. Dietary phyto-oestrogens and the risk of ovarian and endometrial cancers: Findings from two Australian case-control studies. Br. J. Nutr. 2014, 111, 1430-1440. [CrossRef] [PubMed]

24. He, J.; Wang, S.; Zhou, M.; Yu, W.; Zhang, Y.; He, X. Phytoestrogens and risk of prostate cancer: A meta-analysis of observational studies. World, J. Surg. Oncol. 2015, 13, 231. [CrossRef] [PubMed]

25. McCann, S.E.; Hootman, K.C.; Weaver, A.M.; Thompson, L.U.; Morrison, C.; Hwang, H.; Edge, S.B.; Ambrosone, C.B.; Horvath, P.J.; Kulkarni, S.A. Dietary intakes of total and specific lignans are associated with clinical breast tumor characteristics. J. Nutr. 2012, 142, 91-98. [CrossRef] [PubMed]

26. Velentzis, L.S.; Cantwell, M.M.; Cardwell, C.; Keshtgar, M.R.; Leathem, A.J.; Woodside, J.V. Lignans and breast cancer risk in pre- and post-menopausal women: Meta-analyses of observational studies. Br. J. Cancer 2009, 100, 1492-1498. [CrossRef] [PubMed]

27. Sun, Q.; Wedick, N.M.; Pan, A.; Townsend, M.K.; Cassidy, A.; Franke, A.A.; Rimm, E.B.; Hu, F.B.; van Dam, R.M. Gut microbiota metabolites of dietary lignans and risk of type 2 diabetes: A prospective investigation in two cohorts of U.S. women. Diabetes Care 2014, 37, 1287-1295. [CrossRef] [PubMed]

28. Lin, Y.; Yngve, A.; Lagergren, J.; Lu, Y. A dietary pattern rich in lignans, quercetin and resveratrol decreases the risk of oesophageal cancer. Br. J. Nutr. 2014, 112, 2002-2009. [CrossRef] [PubMed]

29. Zaineddin, A.K.; Buck, K.; Vrieling, A.; Heinz, J.; Flesch-Janys, D.; Linseisen, J.; Chang-Claude, J. The association between dietary lignans, phytoestrogen-rich foods, and fiber intake and postmenopausal breast cancer risk: A German case-control study. Nutr. Cancer 2012, 64, 652-665. [CrossRef] [PubMed]

30. Zamora-Ros, R.; Forouhi, N.G.; Sharp, S.J.; González, C.A.; Buijsse, B.; Guevara, M.; van der Schouw, Y.T.; Amiano, P.; Boeing, H.; Bredsdorff, L.; et al. The association between dietary flavonoid and lignan intakes and incident type 2 diabetes in European populations: The EPIC-InterAct study. Diabetes Care 2013, 36, 3961-3970. [CrossRef] [PubMed]

31. Grosso, G.; Stepaniak, U.; Micek, A.; Kozela, M.; Stefler, D.; Bobak, M.; Pajak, A. Dietary polyphenol intake and risk of type 2 diabetes in the Polish arm of the Health, Alcohol and Psychosocial factors in Eastern Europe (HAPIEE) study. Br. J. Nutr. 2017, 118, 60-68. [CrossRef] [PubMed]

32. Wallström, P.; Drake, I.; Sonestedt, E.; Gullberg, B.; Bjartell, A.; Olsson, H.; Adlercreutz, H.; Tikkanen, M.J.; Wirfält, E. Plasma enterolactone and risk of prostate cancer in middle-aged Swedish men. Eur. J. Nutr. 2017. [CrossRef] [PubMed]

33. Nooyens, A.C.; Milder, I.E.; van Gelder, B.M.; Bueno-de-Mesquita, H.B.; van Boxtel, M.P.; Verschuren, W.M. Diet and cognitive decline at middle age: The role of antioxidants. Br. J. Nutr. 2015, 113, 1410-1417. [CrossRef] [PubMed]

34. Zamora-Ros, R.; Knaze, V.; Luján-Barroso, L.; Kuhnle, G.G.; Mulligan, A.A.; Touillaud, M.; Slimani, N.; Romieu, I.; Powell, N.; Tumino, R.; et al. Dietary intakes and food sources of phytoestrogens in the European Prospective Investigation into Cancer and Nutrition (EPIC) 24-h dietary recall cohort. Eur. J. Clin. Nutr. 2012, 66, 932-941. [CrossRef] [PubMed] 
35. Van der Schouw, Y.T.; Sampson, L.; Willett, W.C.; Rimm, E.B. The usual intake of lignans but not that of isoflavones may be related to cardiovascular risk factors in U.S. men. J. Nutr. 2005, 135, 260-266. [CrossRef] [PubMed]

36. De Kleijn, M.J.; van der Schouw, Y.T.; Wilson, P.W.; Adlercreutz, H.; Mazur, W.; Grobbee, D.E.; Jacques, P.F. Intake of dietary phytoestrogens is low in postmenopausal women in the United States: The Framingham study. J. Nutr. 2001, 131, 1826-1832. [CrossRef] [PubMed]

37. Wolk, A.; Bergstrom, R.; Hunter, D.; Willett, W.; Ljung, H.; Holmberg, L.; Bruce, A.; Adami, H.O. A prospective study of association of monounsaturated fat and other types of fat with risk of breast cancer. Arch. Intern. Med. 1998, 158, 41-45. [CrossRef] [PubMed]

38. Milder, I.E.; Feskens, E.J.; Arts, I.C.; de Mesquita, H.B.; Hollman, P.C.; Kromhout, D. Intake of the plant lignans secoisolariciresinol, matairesinol, lariciresinol, and pinoresinol in Dutch men and women. J. Nutr. 2005, 135, 1202-1207. [CrossRef] [PubMed]

39. Penalvo, J.L.; Haajanen, K.M.; Botting, N.; Adlercreutz, H. Quantification of lignans in food using isotope dilution gas chromatography/mass spectrometry. J. Agric. Food Chem. 2005, 53, 9342-9347. [CrossRef] [PubMed]

40. Godos, J.; Bergante, S.; Satriano, A.; Pluchinotta, F.R.; Marranzano, M. Dietary phytoestrogen intake is inversely associated with hypertension in a cohort of adults living in the Mediterranean area. Molecules 2018, 23, 368. [CrossRef] [PubMed]

41. McGill, C.R.; Kurilich, A.C.; Davignon, J. The role of potatoes and potato components in cardiometabolic health: A review. Ann. Med. 2013, 45, 467-473. [CrossRef] [PubMed]

42. Kapusta-Duch, J.; Kopeć, A.; Piatkowska, E.; Borczak, B.; Leszczyńska, T. The beneficial effects of Brassica vegetables on human health. Roczniki Państwowego Zakładu Higieny 2012, 63, 389-395. [PubMed]

43. Sesso, H.D.; Gaziano, J.M.; Liu, S.; Buring, J.E. Flavonoid intake and the risk of cardiovascular disease in women. Am. J. Clin. Nutr. 2003, 77, 1400-1408. [CrossRef] [PubMed]

(C) 2018 by the authors. Licensee MDPI, Basel, Switzerland. This article is an open access article distributed under the terms and conditions of the Creative Commons Attribution (CC BY) license (http://creativecommons.org/licenses/by/4.0/). 